\title{
Caught in the act: Observation of the Solvent Response triggered by Excited-State Proton Transfer in real time
}

\author{
C. Hoberg, ${ }^{1}$ T. Ockelmann, ${ }^{1}$ J. Shee, ${ }^{2}$ P. Balzerowski ${ }^{1}$, D. DasMahanta, F. Novelli ${ }^{1}$, M. Head-Gordon ${ }^{2,3}$ \\ and M. Havenith ${ }^{1 *}$
}

${ }^{1}$ Lehrstuhl für Physikalische Chemie II, Ruhr-Universität Bochum, 44780 Bochum, Germany

${ }^{2}$ Kenneth S. Pitzer Center for Theoretical Chemistry, Department of Chemistry, University of California, Berkeley, California 94720, USA

${ }^{3}$ Chemical Sciences Division, Lawrence Berkeley National Laboratory, Berkeley, California 94720, USA

*Martina.Havenith@rub.de

\begin{abstract}
Real-time observation of the solvent response following Excited State Proton Transfer (ESPT) of the photoacid HPTS into water using Optical Pump THz Probe (OPTP) spectroscopy from $0.1 \mathrm{ps}$ up to $300 \mathrm{ps}$ is reported. Subsequent to an instantaneous $(<0.2 \mathrm{ps})$ electronic response of the solute to photoexcitation, an oscillation with a period of $4 \mathrm{ps}$ involving an intermolecular $\mathrm{H}_{\text {pyranine }} \mathrm{O}_{\text {water }}$ mode is observed. Proton transfer in HPTS starts at $(<0.4$ ps). While for the methylated derivative, MPTS, and the deprotonated photoacid this oscillation relaxes on a time scale of $1.5-2 \mathrm{ps}$, for HPTS the oscillation decays more rapidly within 0.4 ps, which marks the onset of proton transfer. Energy transfer from the excited solute to the solvent takes place on a time scale of 120 ps and is proportional to the Stokes shift associated with energetic relaxation from the Franck-Condon region to the ground state of the photoexcited HPTS.
\end{abstract}

Teaser: Optical Pump-THz Probe spectroscopy enables us to probe the solvent reaction coordinate in real-time, revealing a detailed mechanism of pyranine photoacidity. 


\section{Introduction}

Photo-induced excited-state (ES) proton transfer (PT) reactions are of central importance in many biological and chemical processes, and numerous studies have examined ESPT by a photoacid into a bulk liquid such as water ${ }^{1}$. Ultrafast fluorescence and infrared (IR) techniques have been successfully used to probe the electronic structures or intramolecular vibrations of photoacids or bases as well as reaction intermediates, and the rate constants obtained have enabled the deduction of elementary reaction steps involved in ESPT processes ${ }^{2-11}$. ESPT is suggested to follow a two-step or three-step mechanism ${ }^{5,12}$, the latter involving solvent-controlled reversible dissociation inside the solvent shell followed by diffusion of the proton into the solvent. Previous experimental studies indicated that the initial PT of strong photoacids is controlled by solvent relaxation ${ }^{13,14}$. Indeed, solvent fluctuations and rearrangements are proposed to play essential roles in forming the correct hydrogen bond configuration and solvent polarization to facilitate $\mathrm{PT}^{15}$. Theoretical studies have also underlined the need to track ultrafast structural reorganization of the solvent subsequent to photoexcitation of proteins $^{16-18}$.

In this work, we aim to shed light on the solvent reaction coordinate using a novel set-up for transient Terahertz-optical spectroscopy. We present Optical-Pump THz-Probe (OPTP) spectroscopy (1-3 THz) experiments with sub-ps time resolution and a high dynamic range ( $>70 \mathrm{~dB})$ which allows us to probe the solute-solvent response in photo-induced reactions. The time interval of 300 ps spans initial proton release, the relaxation of the solvent coordinate, and the thermalization of the energy into the solvent. Probing subtle changes of the solvent upon ESPT on a background of bulk solvent dynamics in real time constitutes a major experimental challenge. Terahertz $(\mathrm{THz})$ spectroscopy $(0.1-10 \mathrm{THz})$ is a sensitive tool to probe solvation dynamics and the hydration of ions, amino acids and proteins as well as the formation of Zundel or Eigen cations ${ }^{19-24}$. Ab initio molecular dynamics (AIMD) simulations made it possible to dissect the broad $\mathrm{THz}$ spectra for water and small solutes and identify specific collective intermolecular modes involving the first and even the second hydration shells ${ }^{25-29}$. These $\mathrm{THz}$ fingerprints could be directly correlated to changes in the hydration network ${ }^{30,31}$.

OPTP is a common technique in solid state physics, especially for semiconductors where it is used to probe , e.g., carrier dynamics ${ }^{32,33}$. In contrast, so far very little work was been done on the liquid phase. In 1997, McElroy and Wynne published OPTP results on betaine 30 and p-nitroaniline in $m$-dichlorobenzene solution ${ }^{34}$. In the same year, Haran et al. obtained data on betaine 30 in chloroform ${ }^{35}$. These molecules exhibit a large change in dipole moment upon optical excitation. The studies revealed transient signals with time constants around $1-1.5$ ps which are, in both studies, linked to the reorientation of solvent molecules in the vicinity of the dye molecules and the resulting changes in low-frequency collective motions. In 2003 Kadlec et al. used the OPTP technique to study 
the response of organic solvents to the excitation of two dyes, TBNC and Coumarin $153^{36}$. In the following years, studies on solvated electrons in $n$-hexane and aqueous solution were performed ${ }^{37,38}$. To our knowledge, the first OPTP study in water was carried out in 2018 by Ahmed et al. on coumarin 343 in basic aqueous solution ${ }^{39}$. In their experiment, a 10 ps time constant, which is close to the Debye relaxation time of 8 ps in bulk water ${ }^{40}$, was associated to the dielectric response within the solvation layer of the solute upon electronic excitation. Based on simulations, they predicted a nonequilibrium temperature increase in the first hydration shell upon electronic excitation on time scales of $2-3 \mathrm{ps}^{39}$.

By stabilizing the $\mathrm{THz}$ radiation source and the water jet, such that averaging over more than 12 hours become possible, we were now able to observe the fine details of the solvent response beyond overall "heating" and Debye relaxation processes.

Here, we present the OPTP spectrum of a prototypical photoacid, 8-hydroxypyrene-1,3,6-trisulfonate (HPTS), also known as pyranine. An overview of ESPT in HPTS has been given by Kumpulainen et al ${ }^{12}$. HPTS is highly soluble in water, photostable, and has a quantum yield close to unity ${ }^{41}$. When optically excited $\left(\lambda_{\max }=403 \mathrm{~nm}\right)$, the $\mathrm{pK}_{\mathrm{a}}$ of HPTS drops from 7.2 in the ground state to 0.4 in the excited state ${ }^{2,42}$. Pioneering ps-ns fluorescence studies on the excited state proton transfer of HPTS by Pines and Huppert (starting in the 1980s) yielded time scales of 100 ps for the proton transfer ${ }^{1,3,43}$. Based on the observation of a non-exponential decay for longer time scales and higher concentrations, a diffusion controlled geminate recombination process was proposed. Using femtosecond fluoresecence upconversion spectroscopy Tran-Thi and co-workers found that proton release takes place on a time scale of $87 \mathrm{ps}\left(\text { in } \mathrm{H}_{2} \mathrm{O}\right)^{44}$. UV/IR measurements by Rini et al. confirmed these time scales by observation of the IR-active fingerprint modes ${ }^{5}$. In these experimental studies the dominant charge-transfer pathway is attributed to a sub-ps "through bridge" event in which the bridge adopts an Eigen-like (hydronium) structure. This was confirmed by ab initio Molecular Dynamics studies ${ }^{45}$, while, Siwick and Bakker explained the long time scale of PT in HPTS by Grotthus-like conduction between the acid and the base on a proton wire spanning several water molecules ${ }^{46,47}$. Transient IR absorption spectroscopy allows to follow the proton dissociation of the HPTS photoacid and the concomitant generation of the HPTS photobase, as well as to characterize the transient absorption of the aqueous proton shuttling in between acid and base by observation of the IR proton continuum. However, the exact molecular mechanism of the key role of the solvent still remains to be unraveled ${ }^{48}$.

In summary, fs-ps fluorescence and IR studies probing sensitive changes of the pyranine photoacid or conjugate base yielded three time constants. The shortest time constant of $\sim 0.3$ ps was attributed to a fast solvent relaxation step in the vicinity of the photoacid or an initial charge separation step ${ }^{9,49}$. The longest time constant of 80-100 ps reflects diffusion-assisted proton dissociation ${ }^{12}$. Furthermore, an 
intermediate process with a time constant of 2-3 ps was observed, whose interpretation is debated $^{12,50}$. This intermediate step exhibits distinct spectral features in comparison to the electronically-excited solute or the deprotonated chromophore, and has been assigned to: a) hydrogen bond formation between HPTS and a specific water molecule and/or subsequent reorganization of surrounding water molecules ${ }^{44}$; b) the transition to electronic states possibly involving a slow charge transfer process ${ }^{50-54}$; c) formation of contact ion pairs $(\mathrm{CIP})^{5,13,49,55}$; d) the hydrated proton "in flight" between the acid and the base ${ }^{46,47,56}$. According to Vauthey, unambiguous evidence for one of the above interpretations has yet to be provided ${ }^{57}$.

Due to recent experimental advances in OPTP spectroscopy, we are now able to observe in real time the finer details of the solvent response in HPTS and derivative chromophores. Our results demonstrate the exceptional sensitivity of OPTP, which enables us to follow in detail the response of the solvent, separating out the onset of PT from heat transfer, among other processes.

\section{Results}

\section{Experimental}

The OPTP setup in Bochum has been described before ${ }^{58}$. An overview is given in the methods section of this paper. In short, using a plasma-generated $\mathrm{THz}$ beam as probe beam and a water flat-jet with excellent stability enabling long measurement times (typ. $10 \mathrm{~h}$ ), we could increase the sensitivity to detect changes in the THz field amplitude smaller than $5 \times 10^{-5}$ in liquid samples. To compare the OPTP traces of the photoacid HPTS, we also investigated the HPTS derivative MPTS (8-methoxypyrene-1,3,6trisulfonic acid), which is very similar in electronic structure but does not undergo PT. MPTS is obtained by substitution of the hydroxyl with a methoxy group.

The results of the OPTP traces for HPTS, MPTS and OPTS (the deprotonated HPTS) in $\mathrm{H}_{2} \mathrm{O}$ are displayed in Fig. 1. We plot the change in the peak amplitude of the transmitted $\mathrm{THz}$ electric field $(\Delta \mathrm{E})$ as a function of pump-probe delay $(\mathrm{t})$, normalized to the signal at $\mathrm{t}=0 \mathrm{ps}$, denoted as $\Delta \mathrm{E} / \mathrm{E}$. Both the instantaneous signal ( $<0.2 \mathrm{ps}$ ) as well as the long-lasting response scale linearly with the pump fluence. Note that both the pump as well as the probe are linearly (parallel) polarized. Therefore, isotropic as well as anisotropic changes in absorption or refraction will yield a signal change in the transmitted electric field of the $\mathrm{THz}$ probe. In the pump-probe overlap region a pulse-width limited spike ( $<0.2 \mathrm{ps})$ in the transmitted THz amplitude is observed: $\triangle E / E$ peaks around $14 \times 10^{-3}$ for HPTS, MPTS and OPTS (see Fig. 1). Note that the time resolution is restricted by the instrument response time $(88 \mathrm{fs})^{58}$. This instantaneous signal is attributed to an electronic response, e.g., a change in the solvent polarizability. 

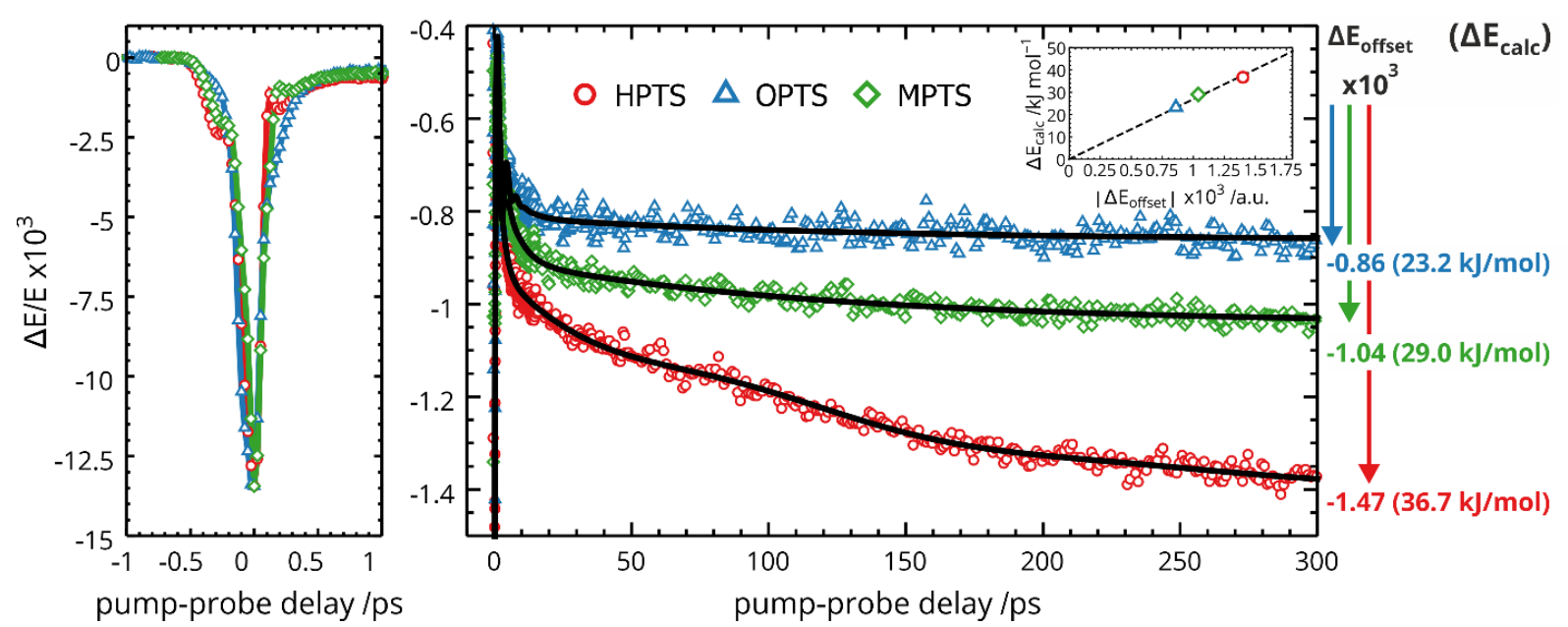

Fig. 1. OPTP signal of the HPTS, OPTS, and MPTS aqueous solutions at pump-probe overlap (left), and the transient OPTP traces out to 300 ps (right). The data shown is filtered using a 2 point moving average. Note that the magnitude of the transient signal beyond pump-probe overlap is one order of magnitude smaller than the signal at time zero. The black lines show the best fits of the global data analysis as discussed in the text. On the right side, we show the amplitude of the negative OPTP offset $\Delta \mathrm{E}_{\text {offset }}$ (see Table 1 ). Next to $\Delta \mathrm{E}_{\text {offset }}$ we also give the calculated Stokes shifts associated with relaxation from the So geometry of the Franck-Condon region of the vertical excitation to the optimal $\mathrm{S}_{1}$ structure $\left(\Delta \mathrm{E}_{\mathrm{calc}}\right)$. Both values are linearly correlated, as shown in the inset (top right).

For pump-probe time delays beyond 1 ps we observe a decrease $(\Delta E / E<0)$, i.e., an increase in $T H z$ absorption. Most notably, $\Delta E / E$ remains negative on a time scale that exceeds our measurement time (300 ps).

While a long lasting ( $>300 \mathrm{ps}$ ) decrease of $\Delta \mathrm{E} / \mathrm{E}$ is observed for all three cases, the normalized change in the transmitted THz field amplitude differs for HPTS, MPTS, and OPTS, as shown in Fig. 1. The magnitude of this offset ( $\left.\Delta \mathrm{E}_{\text {offset }}\right)$ is a signature of the overall energy release into the solvent, as explained later.

In Fig. 2 we zoom into the first 15 ps time interval after photoexcitation for HPTS, MPTS, and OPTS. Surprisingly, beyond the expected long term response, which can be described by a biexponential decay for OPTS and MPTS (Fig. 2 b,c) the initial signal resembles a classical damped harmonic oscillation. For HPTS, the dampening becomes large, such that the oscillation becomes overdamped and resembles an exponential decay (Fig.2a).

We have fitted the three OPTP traces to a sum of one damped harmonic oscillation and a biexponential decay, as described by:

$$
\frac{\Delta E}{E}(t)=S_{D H}(t)+S_{E 1}(t)+S_{E 2}(t)
$$



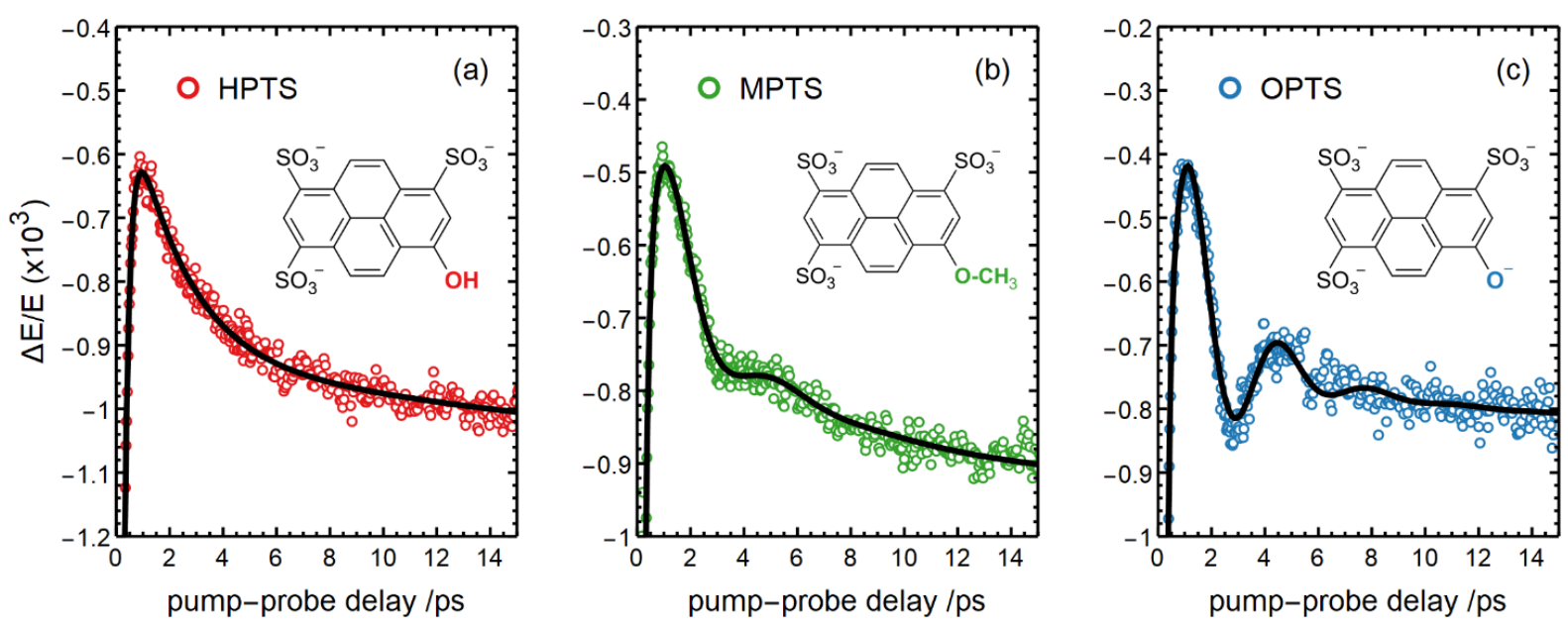

Fig. 2. OPTP signals at pump-probe delays between $0 \mathrm{ps}$ and $+15 \mathrm{ps}$ obtained from $20 \mathrm{mM}$ aqueous solutions of a) HPTS, b) MPTS, and c) OPTS.

In the cases of OPTS and MPTS, the damped harmonic oscillation is expressed as:

$$
S_{D H}(t)=\frac{\mathrm{a}_{0}}{\sqrt{\omega_{0}^{2}-\delta^{2}}} e^{-\delta t} \sin t \sqrt{\omega_{0}^{2}-\delta^{2}}
$$

Here, $\omega_{0}$ and $\delta$ denote the undamped angular frequency and damping constant, respectively. In the case of HPTS, where an overdamped response is observed $\left(\delta>\omega_{0}\right)$, Eq. (2) can be simplified to a sum of two exponential decaying terms:

$$
S_{\mathrm{DH}}(t)=A_{1} e^{-t / \tau_{\mathrm{DH} 1}}+A_{2} e^{-t / \tau_{\mathrm{DH} 2}}
$$

where

$$
\begin{aligned}
& \tau_{\mathrm{DH} 1 / 2}=\left(\delta \pm \sqrt{\delta^{2}-\omega_{0}^{2}}\right)^{-1} \\
& A_{1 / 2}=\mp \frac{\mathrm{a}_{0}}{2 \sqrt{\delta^{2}-\omega_{0}^{2}}}
\end{aligned}
$$

For HPTS, MPTS, and OPTS, the long-term response can be modeled as a bi-exponential decay and a long-lasting offset:

$$
S_{E 1}(t)+S_{E 2}(t)=a_{1} e^{-t / \tau_{1}}+a_{2} e^{-t / \tau_{2}}+\Delta E_{\text {offset }}
$$

While the amplitudes $a_{1}$ and $a_{2}$ varied significantly between MPTS and OPTS, the time constants $\tau_{1}$ and $\tau_{2}$ are similar for all three molecules. For OPTS and MPTS two exponentials with time constants of $\tau_{1}=5.4 \mathrm{ps}$ and $\tau_{2}=120 \mathrm{ps}$ are required to describe the data adequately. 
Table 1. Fit results for the OPTP signal for HPTS, MPTS and OPTS, describing the short time response, the longer term response by an (over)damped harmonic oscillator, and the very long term exponential response $\left(a_{2}\right)$.

\begin{tabular}{cccccccccc}
\hline & ESPT? & $a_{0} \times 10^{3}$ & $\omega_{0} / \mathrm{ps}^{-1}$ & $\mathrm{~T} / \mathrm{ps}$ & $\delta / \mathrm{ps}^{-1}$ & $1 / \delta / \mathrm{ps}$ & $\begin{array}{c}\mathrm{a}_{1} \times 10^{4} \\
\left(\tau_{1}: 5.4 \mathrm{ps}\right)\end{array}$ & $\begin{array}{c}\mathrm{a}_{2} \times 10^{4} \\
\left(\tau_{2}: 120 \mathrm{ps}\right)\end{array}$ & $\begin{array}{c}\Delta \mathrm{E}_{\text {offset }} \\
\times 10^{3}\end{array}$ \\
\hline HPTS & $\boldsymbol{V}$ & 1.75 & 1.52 & 4.1 & 2.6 & 0.38 & 0 & 5.6 & -1.47 \\
MPTS & $\mathbf{X}$ & 0.43 & 1.58 & 4.0 & 0.7 & 1.4 & 3.1 & 1.4 & -1.04 \\
OPTS & $\mathbf{X}$ & 0.70 & 1.91 & 3.3 & 0.5 & 2.0 & 1.7 & 0.5 & -0.86 \\
\hline
\end{tabular}

For HPTS the amplitude $a_{1}$ was zero within our experimental uncertainty. In the case of HPTS, the overdamped oscillation $\left(S_{\mathrm{DH}}\right)$ yields an overall biexponential decay with time constants of $\tau_{\mathrm{DH} 1}=0.2 \mathrm{ps}$ and $\tau_{\mathrm{DH}_{2}}=2 \mathrm{ps}$. All traces show a long-lasting plateau for which a constant $\Delta \mathrm{E}_{\text {offset }}$ is included. The result of the fit is summarized in Table 1.

Interestingly, for HPTS and MPTS the fitted undamped angular frequencies $\omega_{0}$ of $\sim 1.52 \mathrm{ps}^{-1}$ and 1.58

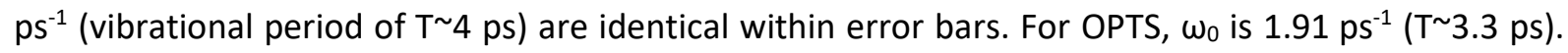
While the oscillation period is very similar, the damping constant increases when PT occurs, accounting for the different responses detected in the first 15 ps (see Fig. 2). The largest damping constant was found for HPTS $\left(\delta>\omega_{0}\right)$, while for MPTS and OPTS $\delta<\omega_{0}$ still holds.

\section{Computational}

In this section, we utilize electronic structure calculations in an effort to indirectly elucidate the solvent response to photoexcitation. Time-dependent density functional theory (TDDFT) calculations were performed to characterize the changes in electron density upon photoexcitation for all three solutes, and the resulting attachment/detachment density plots are shown in Fig. 3.

In HPTS, MPTS, and OPTS, the electronic transition to the first excited state shows two main features. First, there is significant rearrangement of charge within the pyrene ring system, which leads to changes in the geometric structure of $S_{1}$ versus $S_{0}$. The Stokes shifts associated with relaxation from the $S_{0}$ geometry of the Franck-Condon region of the vertical excitation to the optimal $S_{1}$ structure are calculated to be $23.2,29.0$, and $36.7 \mathrm{~kJ} / \mathrm{mol}$ for HPTS, MPTS, and OPTS. The ordering of these magnitudes is consistent with that of the observed damping constants as well as the long-lasting offset $\Delta \mathrm{E}_{\text {offset. }}$. This energy will be dispersed into solute modes upon excitation, and will then be transmitted in due course to solvent degrees of freedom as well. 
HPTS

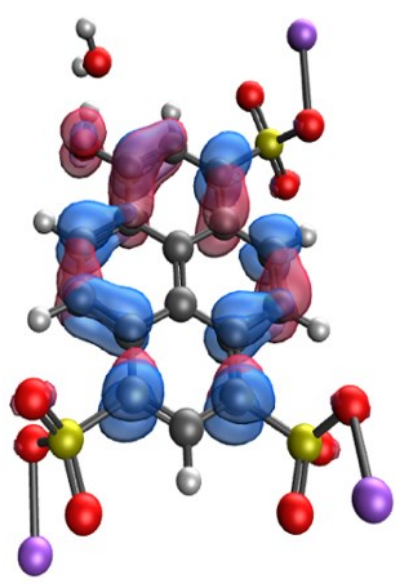

MPTS

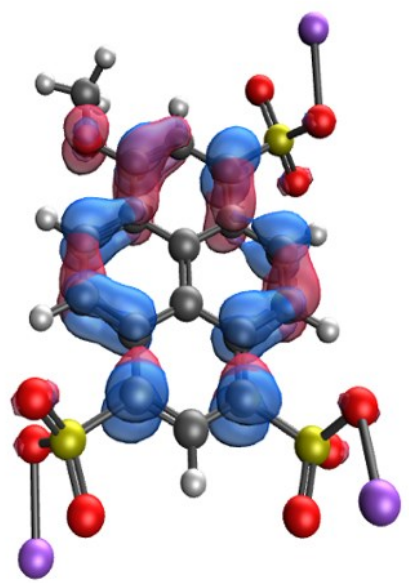

OPTS

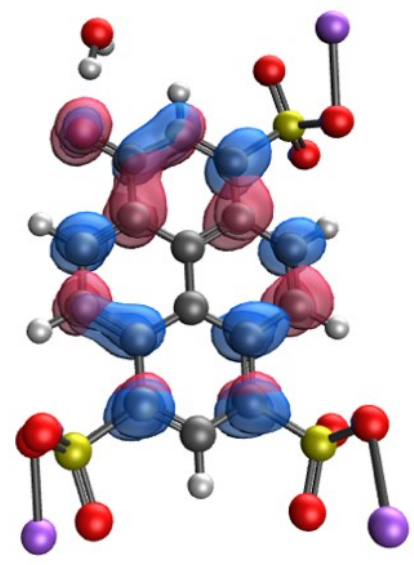

Fig. 3. Attachment (blue) and detachment (red) densities corresponding to vertical excitation of HPTS, MPTS, and OPTS.

From Figure 3, one can also observe that part of the calculated electron density change upon excitation involves the removal of a fraction of an electron from the oxygen atom of the $\mathrm{OH}, \mathrm{OMe}$, and $\mathrm{O}$ functional groups and its redistribution into the $\pi$-system of the pyrene ring. This provides evidence for a mesomeric effect in which electron density corresponding to the lone pair in an atomic $p$ orbital of the oxygen atom is donated into the neighboring $\pi$-system, i.e. the pyrene scaffold. That such an effect occurs spontaneously in the excited state can be understood in the context of Baird's rule of (anti)aromaticity ${ }^{59}$, which states roughly that a cyclic aromatic molecule with $4 \mathrm{~N}+2 \pi$ electrons in its perimeter will be aromatic (and energetically stabilized) in the ground-state but anti-aromatic (and energetically destabilized) in the excited state. The opposite holds for such systems with $4 \mathrm{~N} \pi$ electrons, which will be anti-aromatic in the ground state and aromatic in the excited state. This has recently been observed spectroscopically ${ }^{60}$, and has been used to rationalize ESPT processes. ${ }^{61}$

The substantial Stokes shifts constitute the driving force behind the observed energy transfer to the solvent environment, via energy transfer into solvent vibrational modes for all three species. The density distributions shown in Fig. 3 suggest that these vibrational modes involve a collective motion of the pyrene ring of the solute and surrounding solvent molecules in the first solvation shell. A critical observation from Fig. 3 is that the electronic rearrangements are qualitatively similar in all three species. However, the redistributions that occur in HPTS and MPTS are nearly identical, and differ slightly from that predicted in OPTS. In the latter case, the alternating regions of added and removed densities are more localized ( atom centered), and a larger amount of density appears to be lost at the oxygen atom. This is consistent with previously measured changes in dipole moment upon photoexcitation of 4.2 and 5.2 Debye in HPTS and OPTS, respectively. ${ }^{62}$ The relatively larger 
stabilization of the excited state of OPTS vs that of HPTS, due to stronger interactions with the polar solvent and a greater degree of aromatic character, provides further insight into the photoacidity of this species.

Finally, we note that the calculated bond length between HPTS and an explicit water in the separately optimized ground $\left(S_{0}\right)$ and excited $\left(S_{1}\right)$ states decreases from 1.62 to 1.57 Angstroms, respectively.

\section{Discussion}

Based on previous studies, the following three steps have been discussed: an instantaneous step (<0.1ps), an intermediate step (ca. 2-3 ps) and diffusion limited proton transfer (80-90 ps) $5,12,51,63$.

The instantaneous signal change in the OPTP transient $(<0.1 \mathrm{ps})$ is attributed to the electronic response subsequent to photoexcitation. Nuclear motions such as the nuclear rearrangement of the photoacid or the solvent molecules in the hydration shell will require longer time scales of $>1 \mathrm{ps}$.

The time scale between 1 and 10 ps is characteristic of (longitudinal) relaxation of the first solvation shell. Using OPTP we observe an initial amplitude oscillation when monitoring the transmitted $\mathrm{THz}$ amplitude for OPTS, MPTS, and HPTS (i.e., even in the absence of PT, see Fig. 2). For both HPTS and MPTS, the period, $T$, of this oscillation is $\sim 4.0-4.1 \mathrm{ps}$; for OPTS, it is slightly shorter (T $\sim 3.3 \mathrm{ps}$ ). OPTP is most sensitive to signals in the frequency range below $2 \mathrm{THz}\left(67 \mathrm{~cm}^{-1}\right)$, which include translational and so-called rattling modes, i.e. THz signature of the motion of a charge in/with its hydration shell ${ }^{23,27}$. Using AIMD, Chiariello and Rega predicted the low frequency spectrum of the solvated photoexcited HPTS- The intermolecular $\mathrm{H}_{\text {pyr }}-\mathrm{O}_{\text {water }}$ stretch mode has an absorption in the frequency range below $100 \mathrm{~cm}^{-1} .64$ Thus, we propose, that subsequent to photoexcitation a low-frequency nuclear displacement is triggered that involves the skeleton motion of the photoacid against the first hydration shell. The signal is observed independently of $\mathrm{PT}^{50,51}$. However, for HPTS, the intensity is a factor of 4 larger compared to MPTS and OPTS, which do not exhibit PT ( $a_{0}$, see Table 1). This is in line with our previous $\mathrm{THz}$ studies which revealed a significant increase in intensity depending on the protonation state, e.g., the intensity of the N-C-C-O mode of the glycine zwitterion is increased by $80 \%$ compared to the neutral glycine ${ }^{25}$.

The 4 ps oscillation corresponds to a frequency of $8 \mathrm{~cm}^{-1}$. Based on the well-known frequencies of the rattling modes of anions, we can estimate the frequency range expected for pyranine: The rattling mode of $\mathrm{Cl}^{-}$, more specifically, the self-term of the $\mathrm{Cl}^{-}$ion and its cross-correlations with water molecules in the first and second shell is centered at $200 \mathrm{~cm}^{-1}$, as was shown in a joint experimental and theoretical study ${ }^{27}$. When we take into account that the square root of the mass ratio of HPTS to $\mathrm{Cl}^{-}$is 524/35 and assume a reduction of the force constant in the case of photoexcited HPTS, we expect 
a frequency on the order of approx. $10 \mathrm{~cm}^{-1}$ for a rattling or "breathing" mode, in agreement with our observation.

As shown in Fig. 3, both HPTS and MPTS show almost an identical instantaneous change in charge density. Interestingly, both have the same oscillation period (4 ps) independent of whether or not PT occurs, which suggests that this oscillation is triggered by the change in charge distribution. A slightly different response upon electronic excitation is predicted for OTPS (Fig. 3). We note that the observed oscillation period for OTPS is close to, but still distinct from, that of HPTS and MPTS. We did not observe any change in the oscillation period upon deuteration, i.e., when exchanging $\mathrm{H}_{2} \mathrm{O}$ with $\mathrm{D}_{2} \mathrm{O}$ for HPTS solutions (see SI for details). The lack of any isotope effect is in line with the proposed assignment to an intermolecular stretch or translational mode ${ }^{26}$. However, this observation rules out modes involving any hindered rotation of water molecules (e.g. a librational mode).

We note that neither the vibrational period, $\mathrm{T}=4 \mathrm{ps}$, nor the damping time of the initial oscillation, $\tau=1 / \delta=0.38 \mathrm{ps}$, corresponds to the time constant of $2-3 \mathrm{ps}$ reported in previous studies ${ }^{13,49}$. In fact, based on our experiments we provide an alternative interpretation: The previously observed intermediate time constant for HPTS of 2-3 ps describes the convolution or the envelope of the overdamped oscillation. The damping constant in case of HPTS is 0.4 ps, i.e. almost one order of magnitude faster than previously suggested (see $1 / \delta$ in Table 1 ).

In the absence of PT, e.g for the methylated and deprotonated photoacid, the relaxation of the initial oscillation (Fig. 2) takes place on a typical time scale of $1 / \delta=1.3-1.9$ ps (Table 1 ). This time scale is in agreement with previous values predicted for energy release into the first hydration shell ${ }^{39,65,66}$. It is interesting to note that the same time constant was also observed for MPTS in a transient absorption study: Fang et al. observed an exponential in the center wavelength shift with a time constant of $1.2 \mathrm{ps}$ for MPTS in $\mathrm{H}_{2} \mathrm{O}$, which was attributed to a solvent rearrangement known as longitudinal relaxation. In other words, this describes the relaxation of the coordinated process between the solute and the first hydration shell ${ }^{67}$. Therefore, the observed damping, or exponential decay times of 1.3-1.9 ps, in the absence of PT is attributed to the dissipation of energy into the first hydration shell only. This damping time is considerably faster for HPTS ( 0.4 ps). Thus, the later must be attributed to a different process, e.g. the onset of deprotonation which is consequently proposed to be a time scale of $<0.5 \mathrm{ps}$.

Between 5 and 300 ps we observe for MPTS and OPTS a bi-exponential decay with a time constant of 5-6 ps and a longer time constant of 120 ps. The 5-6 ps exponential response is on the same order of magnitude as the 10 ps response reported by Hamm et al..$^{39}$ for coumarin 343 , and the time constant $\tau_{D}=8.2$ ps Debye relaxation of bulk water ${ }^{68}$. Thus, we attribute the time constant of 5-6 ps to the macroscopic dipole relaxation, which is probed in the low frequency range. 
The slower decrease in the transmitted $\mathrm{THz}$ amplitude (or increase in $\mathrm{THz}$ absorption) on a time scale of $\tau_{2} \sim 120 \mathrm{ps}$ is the time scale of the net energy transfer into the bulk solvent, i.e., the "heating" of the solvent, which is assumed to be diffusion limited. This time scale is the same for MPTS, OPTS, and HPTS. The fitted amplitude $\Delta \mathrm{E}_{\text {offset }}$ differs, and is proportional to the total amount of energy that is deposited into the solvent: $\Delta \mathrm{E}_{\text {offset }}$ is found to be linearly correlated to the calculated Stokes shifts associated with relaxation from the $S_{0}$ geometry of the Franck-Condon region of the vertical excitation to the optimal $\mathrm{S}_{1}$ structure (see inset of Fig 1). The time scale of 120 ps implies that even after 300 ps thermalization has not yet been fully reached, as seen in the raw data.

In $\mathrm{D}_{2} \mathrm{O}$, the energy transfer is observed to be slowed down, with $\tau_{2}=120$ ps in $\mathrm{H}_{2} \mathrm{O}$ compared to $\tau_{2}=150 \mathrm{ps}$ in $\mathrm{D}_{2} \mathrm{O}$ (see the $\mathrm{SI}$ for details). Thus, the measured kinetic isotope effect (KIE) is $150 \mathrm{ps} / 120 \mathrm{ps}=1.17$, in line with an energy transfer either via Debye relaxation or via diffusion. For Debye relaxation, we expect a KIE of 1.25 . The ratio of deuteron to proton mobilities in pure $\mathrm{D}_{2} \mathrm{O}$ and $\mathrm{H}_{2} \mathrm{O}$ is $1.4^{46}$, the isotope ratio of self-diffusion coefficients is $1.22^{69}$.

In the presence of PT transfer, as in HPTS, we find an approximately 5-fold increase in the experimentally observed damping compared to MPTS and OPTS ( $1 / \delta=0.38$ ps for HPTS). In previous studies, a similar time scale ( $0.5 \mathrm{ps}$ ) has been reported for the decay of transient low-frequency modes assigned to ring deformation of photoexcited HPTS by Resonance Raman spectroscopy ${ }^{63}$. Furthermore, in the AIMD simulations by Chiariello and Rega a time constant of 0.5 ps was predicted for the completion of the main nuclear relaxation ${ }^{64}$.

As indicated computationally, upon photoexcitation the pyranine- $\mathrm{H}_{2} \mathrm{O}$ hydrogen bond distance decreases, forming a tighter proton donor-acceptor couple. A decrease in this intermolecular distance and an excitation of an intermolecular pyranine- $\mathrm{H}_{2} \mathrm{O}$ stretch will promote the rapid onset of PT.

We attribute the time scale of 0.4 ps to the time before the onset of proton transfer which is facilitated by a decrease in the intermolecular pyranine- $\mathrm{H}_{2} \mathrm{O}$ hydrogen bond. Thus, based on our results, we conclude that the time scale for transfer into a charge transition state or the onset of protonation is < $0.5 \mathrm{ps}$ and not $2-3 \mathrm{ps}$.

In $\mathrm{D}_{2} \mathrm{O}$ we observe the same oscillation with a damping time constant of $0.4 \mathrm{ps}$, as expected for a triggering via an intermolecular stretch mode. Due to the lack of any isotope effect we can exclude a tunneling PT process. Instead, we propose an adiabatic PT, as in the case of $\mathrm{HCl}$ dissociation, where Ando and Hynes proposed that the proton wave-packet motion will follow the modulation of the proton potential from the reactant solvent configuration to the product state ${ }^{15}$. 

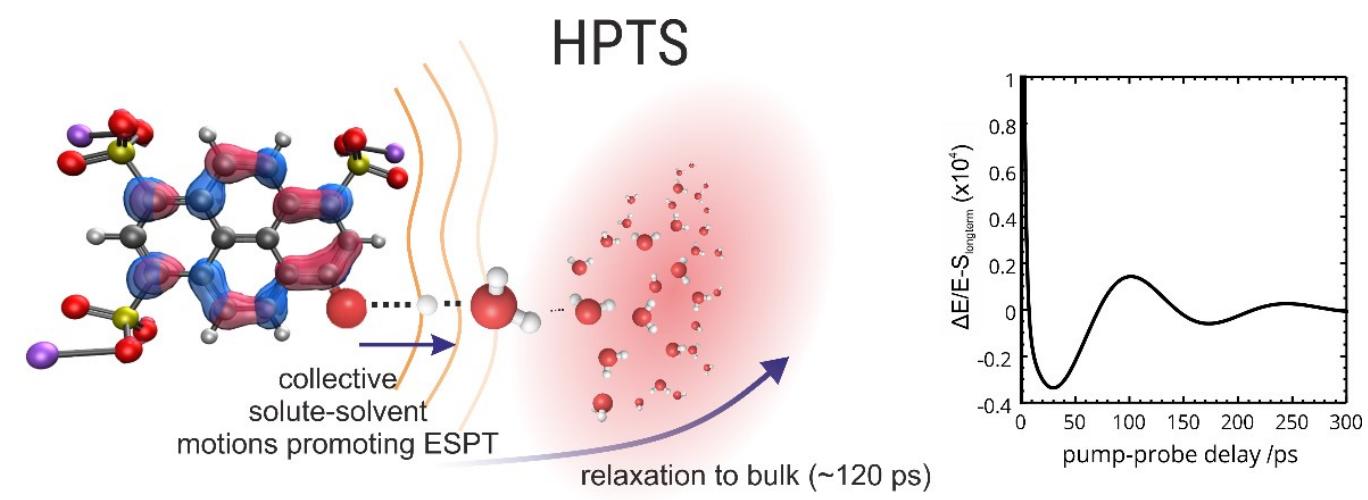

\section{OPTS/MPTS}
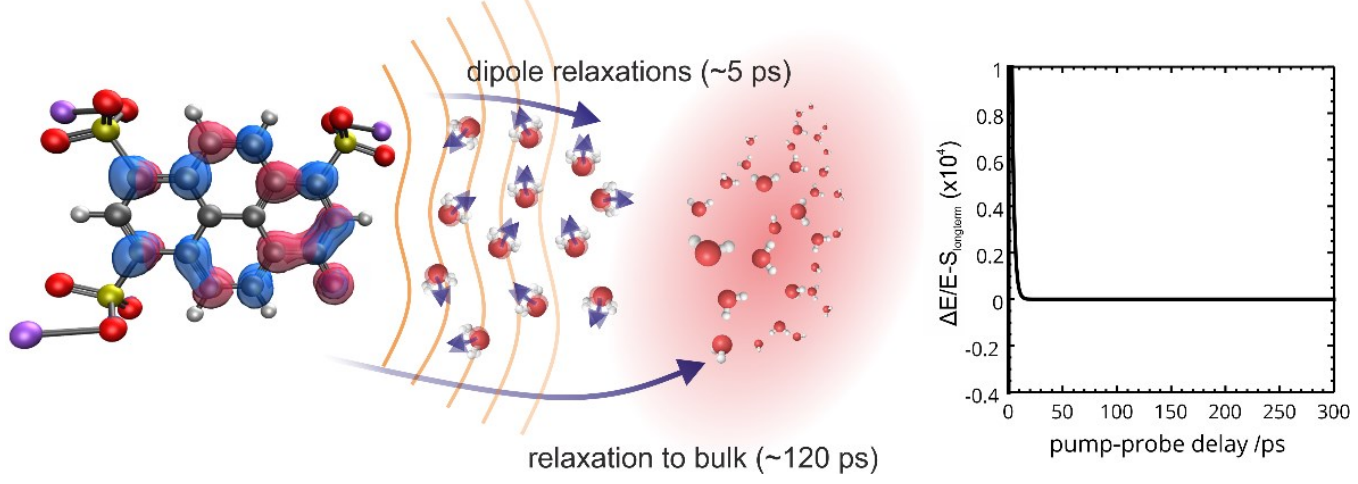

Fig. 4. Left: Response of HPTS and OPTS/MPTS subsequent to electronic excitation. In both cases we observe the initial excitation of an intermolecular collective vibration of solute and solvent. The pyranine- $\mathrm{H}_{2} \mathrm{O}$ hydrogen bond distance decreases, promotes PT and an efficient coupling to the first hydration shell. For OPTS and MPTS the energy release occurs via dipole relaxation, before the energy dissipation is equilibrated into the solvent. Right: We plot the amplitude of the OPTP signal after subtraction of the long term exponential decay ( $\Delta \mathrm{E} / \mathrm{E}-\mathrm{S}$ longterm) which is attributed to the total energy transfer in the solvent. While for OPTS/MPTS the long-term response can be exclusively attributed to the energy transfer or "heat", for HPTS we find a long-term amplitude modulation which is reporting on the proton transfer.

Probing the solvent response during PT. The long-term response of the OPTP signal is dominated by the energy transfer into the solvent described by an exponential decay with a characteristic time scale of $\tau_{2} \sim 120$ ps. In Fig. 5, we plot the OPTP signals of HPTS and MPTS on a logarithmic scale. The longterm exponential decay $\left(\tau_{2}\right)$ is shown as a blue-dashed line. While for MPTS we find a perfect agreement, for HPTS we observe small but systematic deviations (indicated by the arrows) from the exponential decay. This is clearly visible around $100 \mathrm{ps}$, where we observe a small maximum in $\Delta \mathrm{E} / \mathrm{E}$. In order to distinguish the statistical significance of these small deviations from the noise, we repeated the measurement several times. The results were found to be reproducible. 


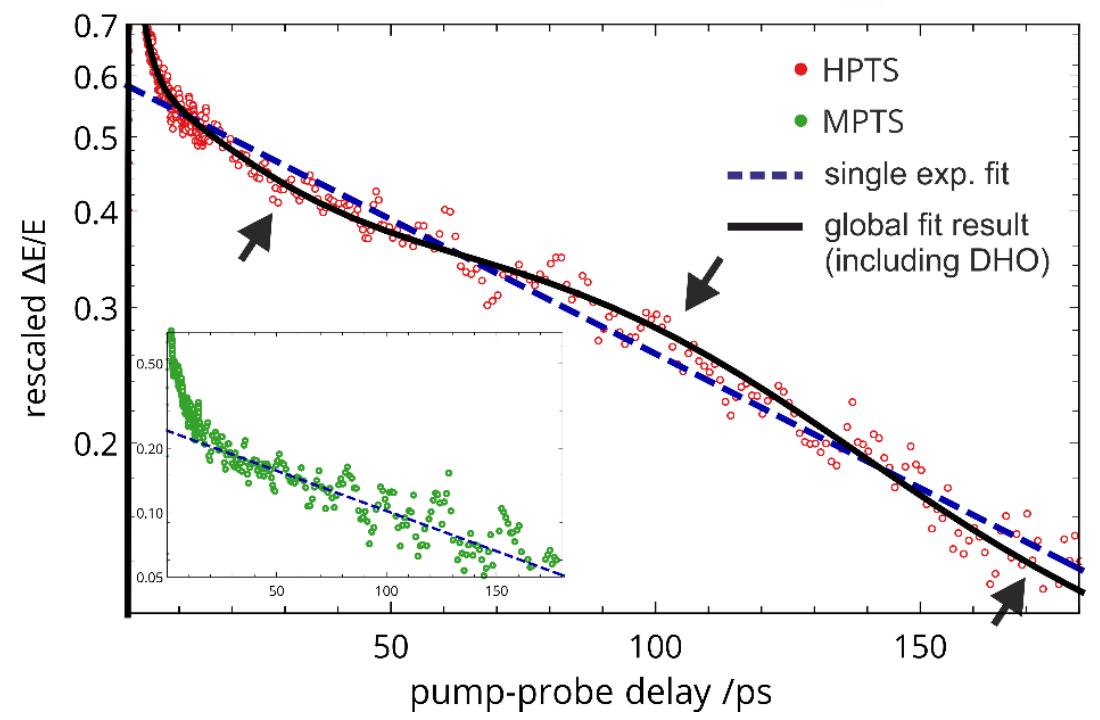

Fig. 5. Logarithmic representation of the HPTS (red) and MPTS transient signals (inset, green). For HPTS the experimental data deviate from a single exponential fit (blue dashed lines) as indicated by black arrows. In contrast, for MPTS no significant deviations are observed (inset). The black curve corresponds to a fit including an additional low-frequency damped harmonic oscillation, see text for details.

Based on these results, we tried to include in the fit a second damped harmonic oscillation $\mathrm{S}_{\mathrm{DH} 2}$ (eq. 2). The fit yielded a vibrational period, $T_{2}$, of $140 \mathrm{ps}$. The damping constant, $\delta_{2}$, is $0.012 \mathrm{ps}^{-1}$, which corresponds to a time constant of $1 / \delta_{2} \sim 83 \mathrm{ps}$. This spectroscopic feature is lacking for MPTS and OPTS, i.e., in cases where no PT takes place. The time constant for the $1 / \delta_{2}$ damped oscillation agrees within error bars with the 90 ps proton diffusion time into the bulk, as reported previously ${ }^{52}$.

First we estimated whether at our experimental conditions, i.e. at a solute concentration of $20 \mathrm{mM}$, an additional process, the diffusion-assisted, reversible geminate-recombination of the proton as proposed by Pines and Huppert ${ }^{70}$ needs to be taken into account: In $\mathrm{D}_{2} \mathrm{O}$ the rates for PT into the solvent $\left(k_{w}\right)$ and the back reaction $\left(k_{g e m}\right)$ are found to be of the same order of magnitude $\left(k_{w}=245 p s s^{-1}\right.$ and $\left.\mathrm{kgm}_{\mathrm{gem}}=900 \mathrm{ps}^{-1}\right)$, however PT into the solvent network still dominates ${ }^{46,56}$.

Previously, it was found that the $\mathrm{H}$-bond network of water supports propagation of phonon-like modes ${ }^{71-73}$. Here we propose that, due to the charge, we can follow the propagating into the bulk solvent, causing an amplitude modulation in $\triangle \mathrm{E} / \mathrm{E}$. On the right side of Fig. 4 we plot the amplitude of $\Delta E / E$ after subtracting the overall exponential decay as a function of time. It should be noted that the damping of an acoustic phonon can be described as exp(- $\alpha r)$, with $r$ being the traveling distance. $\alpha$ scales quadratically with the frequency, i.e. $\alpha / v^{2}=19 \times 10^{-15} \mathrm{sec}^{2} / \mathrm{m}$ at $30^{\circ} \mathrm{C}^{74}$. At a frequency of 10 $\mathrm{GHz}$, the damping is $\alpha=1.9 \times 10^{6} \mathrm{sec}^{2} / \mathrm{m}$. Thus, after propagating even a distance of approx. 300 Angstrom, the damping amounts to only a factor of $\exp (-\alpha r)=0.94$. This rationalizes the observation of a propagation of a low frequency phonon-like wave. 
By assuming a continuum model for solvation, a spherical non-polarizable solute molecule, and a single Debye process of the solvent, we find the following relation between the longitudinal relaxation time, $\tau_{s}$, which involves reorientation only in the first shell, and the Debye relaxation time of a fully solvated dipole in a spherical cavity filled with a uniform, $\tau_{D}{ }^{75,76}$.

$$
\tau_{S}=\left(2 \varepsilon_{\infty}+1\right) /\left(2 \varepsilon_{0}+1\right) \tau_{D}
$$

Here $\varepsilon_{\infty} \sim 1.8$ is the dielectric constant from only the electronic polarizability, and $\varepsilon_{0} \sim 80$ also includes orientational polarizability. For water solvent, this implies that the macroscopic (total dipole) reorientation is $\sim 35 x$ slower than that of a solute-solvent relaxation in the first solvation shell. After the creation of a macroscopic reaction field, the electric field of the dipole is screened by molecules beyond that layer ${ }^{39}$. For time scales of ca. 1 ps, the OPTP experiment probes the solute-solvent response restricted to the first shell, whereas for longer times scales (10-100 ps), i.e. after proton release, we probe the reaction field. While these considerations were derived only for the orientational relaxation time - not including translational modes - we want to point out here that the observed time scale of the second oscillation ( $T=140 \mathrm{ps}$ ) is a factor of 35 increased compared to that of the initial oscillation ( $\mathrm{T}=4 \mathrm{ps})$.

At 100 ps, we find a local increase compared to an exponential decay in the transmitted THz field (see Fig. 5). In the time between 0.5 ps and 100 ps, we expect a change in the protonation state of the solute, from a photoacid to a photobase. In previous THz studies, a protonated solvated solute showed an increase in $\mathrm{THz}$ absorption compared to the neutral solute, while the negatively charged amino acid had a decreased absorption in the frequency range up to $3 \mathrm{THz}^{77}$. If we assume that the same holds for HPTS, then any transformation from photoacid to photobase is expected to result in a relative decrease in absorption, i.e. in a relative increase in transmission.

Final Remarks. ESPT processes can involve several steps occurring at different timescales: photoacid electronic rearrangement (subfs-fs), intrinsic PT (subps-ps), product stabilization, diffusion, and recombination (ps-ns). We report here the direct, real-time observation of the solvent response following Excited State Proton Transfer (ESPT) of the photoacid HPTS into water using Optical Pump THz Probe (OPTP) spectroscopy over a wide range of energy and time scales (from 0.1 ps up to 300 ps). These OPTP experiments enabled us to follow the solvent response after photoexcitation between 0.2 ps and 300 ps in real time, as the wave-packet evolves out of the Frank-Condon zone.

For the photoacid HPTS, the initial rise of the signal is instantaneous (within our experimental time scale). We observe a fast, initial response $(<0.2 \mathrm{ps})$ attributed to the response of the redistribution of electron density on the photoacid. 
In previous studies of HPTS, it was proposed that PT is assisted and modulated by low-frequency modes of solvent molecules in close proximity to the proton-accepting molecule ${ }^{78}$, e.g. a low frequency mode at $180 \mathrm{~cm}^{-1}$ was thought to set the stage for ESPT ${ }^{63}$. The authors speculated that the proton might shuffle back and forth between the photoacid and water molecules within the first solvation shell ${ }^{63}$. In the present paper, we indeed observe an oscillation with an oscillation period of $4 \mathrm{ps}$, which can be attributed to a collective solute-hydration mode independent of PT.

In the Eigen-Weller model for ESPT to solvent the intermediate step is attributed to a reversible shortrange proton transfer from the protonated form to the solvent: $\mathrm{ROH}^{*} \rightleftharpoons\left[\mathrm{RO}^{-*} . . . \cdots \mathrm{H}^{+}\right] \rightleftharpoons \mathrm{RO}^{-*}+\mathrm{H}^{+}$. For the proton transfer of HPTS into water we find no spectroscopic evidence for the formation of a reaction intermediate such as a contact ion pair (CIP). Instead, the same initial oscillation is observed even when PT does not take place, i.e. for MPTS and OPTS. This is in line with Kumpulainen et al. who argued that the CIP* intermediate, if present, might be extremely short-lived and hence thermodynamically unfavorable or bypassed by a Grotthuss-type proton-hopping mechanism ${ }^{79}$. Our experimental results are in line with a two-step mechanism, see Siwick and Bakker ${ }^{46}$, or to a small number of solvent (water) molecules ultimately leading to proton transfer ${ }^{48}$. For HPTS, OPTP spectroscopy we observe an amplitude modulation with a second, local maximum in transmission, with a small amplitude (reduced by a factor of 1000 compared to the initial 4 ps oscillation).

Our OPTP data reveal an overall exponential decrease in the transmitted THz amplitude of the probe beam, which is centered around $1 \mathrm{THz}$, with a time scale of 120 ps. This time constant is consistent with the time scale of the decrease in amplitude of a $420 \mathrm{~cm}^{-1}$ band (110 ps), as observed by Femtosecond Stimulated Raman Spectroscopy ${ }^{63}$. Although the time scale for energy release is close to that for PT (80-90 ps), our study clearly reveals that the 120 ps process describes the diffusion limited energy release into the solvent. The amplitude of the 120 ps offset, i.e. the total energy release into the solvent, is directly proportional to the Stokes shifts associated with relaxation from the FranckCondon region to the minimum $\mathrm{S}_{1}$ structure. In this sense, the $\mathrm{THz}$ probe effectively acts as a " $\mathrm{THz}$ thermometer" ${ }^{39,80}$.

Our results demonstrate the exceptional sensitivity of the present OPTP technique, which enables us to follow the solvent response and the propagation of the charges into the bulk solvent. Thus, this technique allows us to go beyond the solute perspective and map the effect of the reaction on the surrounding solvent to reveal details that were partly or even fully hidden when focusing solely on observables tagged to the photoacid or its conjugate base. 


\section{Materials and Methods}

\section{Experimental}

In Bochum we have built up an OPTP spectrometer with a time resolution of $90 \mathrm{fs}$ and a sensitivity for measuring relative changes in electric field amplitude better than $5 \times 10^{-5} 81$. This allows to follow the ultrafast solvent rearrangement, in real time.

An overview of the optical setup is given in Figure 6. An amplified Ti:Sapphire laser source (SolsticeAce, SpectraPhysics) was used, providing $45 \mathrm{fs}$ pulses centered around $800 \mathrm{~nm}$ with an energy of $1.2 \mathrm{~mJ}$ per pulse at a repetition rate of $5 \mathrm{kHz}$. About $20 \%$ of the energy was used for the optical pump pulse. The pump pulse was frequency-doubled in a $\beta$-BBO crystal (thickness: $200 \mu \mathrm{m}$ ) yielding pulses centered at $400 \mathrm{~nm}$. Another $75 \%$ of the pulse energy was used to generate the $\mathrm{THz}$ probe pulse. This pulse was focused through a $100 \mu \mathrm{m}$ thick $\beta$-BBO crystal and a true zero-order dual-wavelength waveplate $\left(0^{\circ}\right.$ at $400 \mathrm{~nm}, 90^{\circ}$ at $800 \mathrm{~nm}$ ) into air. At the focus, the fundamental and second harmonics produce an airfilament that emits $\mathrm{THz}$ radiation with a conical profile and linear polarization (see supplementary material). The process of $\mathrm{THz}$ emission from two-color filaments has been described in detail before ${ }^{82-}$ 84. In our experiment, we used two-color air-filamentation to generate short ( $190 \mathrm{fs}$ full-width at half maximum, FWHM), broadband THz probe pulses spanning 0.5-10 THz $\left(17-334 \mathrm{~cm}^{-1}\right)$ with an intensity maximum around 1-2 THz $\left(33-67 \mathrm{~cm}^{-1}\right)$. The THz probe-pulse energy was measured to be $10 \mathrm{~nJ}$ (Ophir RM9-THz power meter). $90^{\circ}$ off-axis parabolic mirrors in an 8 -geometry were used to collimate and focus the $\mathrm{THz}$ beam. The THz beam path was purged with nitrogen gas to reduce absorption by water vapor.

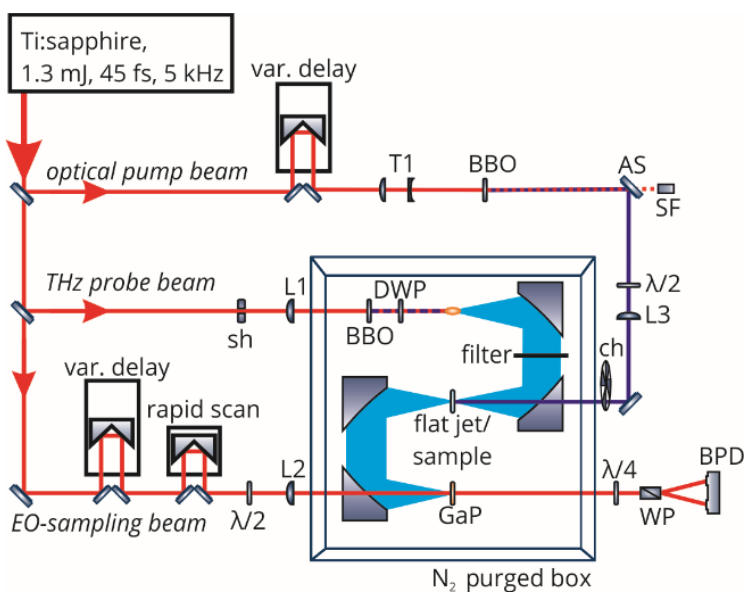

Fig. 6. Overview of the optical setup. BBO: $\beta$-barium borate crystal, DWP: true zero-order dual-wavelength waveplate, SH: shutter, AS: dichroic mirror, GaP: gallium phosphide crystal, WP: Wollaston prism, BPD: balanced silicon photodetectors. 
A low-pass filter after the first parabolic mirror was used to clean the $\mathrm{THz}$ pulse from any residual optical radiation. The optical pump pulse and $\mathrm{THz}$ probe pulse were collinearly overlapped in a freeflowing, flat liquid jet sheet with a thickness of about $100 \mu \mathrm{m}$. This avoids any artifacts related to cuvette window materials and ensures a fresh sample for every laser pulse. Furthermore, it prohibits local heating effects due to the fast flow rate. All experiments were performed at $22^{\circ} \mathrm{C}$. The center of the flat-jet sheet was carefully positioned at the focus of the $\mathrm{THz}$ beam.

The THz E-field was detected via electro-optic sampling in a double-layer $200 \mu \mathrm{m}$ thick $<110>$ gallium phosphide (GaP) crystal contacted with a $1 \mathrm{~mm}<100>\mathrm{GaP}$ crystal, using either step-scanning or a rapid-scanning technique ${ }^{58}$. The optical pump-pulse was chopped, and the difference in the THz E-Field amplitude between the pump-on and pump-off states was recorded at different pump delays. The transient signal obtained at each pump-probe delay was averaged over 2500 pulses. Per measurement around 100 OPTP traces were averaged (10-20 $\mathrm{h}$ measurement time) to obtain a signal to noise (SNR) of $\Delta E / E$ better than $5 \times 10^{-5}$. The instrument time resolution is $88 \mathrm{fs}$, as determined by recording the $\mathrm{THz}$ response upon optical excitation of electron-hole pairs in a high-resistivity silicon sample ${ }^{85}$. In order to exclude systematic errors due to heating or ionization, we verified that no signal was observed in case of a bulk water sample.

HPTS was obtained from ACROS organics. 8-Methoxypyrene-1,3,6-trisulfonate (MPTS) was synthesized from HPTS following a previously reported route ${ }^{86}$. The water was of ultrapure quality with a measured conductivity of less than $0.055 \mu \mathrm{S} / \mathrm{cm}$. Sample concentrations were kept at $20 \mathrm{mM}$. At this concentration no deviation from a highly diluted sample should be expected ${ }^{7}$.

\section{Computational}

Electronic structure calculations were performed with the Q-Chem 5.2 package $^{87}$, and utilize the B3LYP functional ${ }^{88}$, def2-SV(P) basis set ${ }^{89}$, and the polarizable continuum model $(\mathrm{PCM})^{90}$ with a dielectric constant of 78.39 to implicitly account for the effects of water solvent on the electronic structure. For HPTS and OPTS, we include a single water molecule near the $\mathrm{OH}$ or $\mathrm{O}$ - functional groups, respectively, to explicitly account for the intermolecular hydrogen bond. As shown in the Supporting Information, this level of theory produces predictions in quantitative agreement $(\sim 0.1 \mathrm{eV})$ with experimental absorption and fluorescence spectra for these molecules. Linear-response time-dependent density functional theory (TDDFT) within the adiabatic approximation was employed to model excited electronic states ${ }^{91,92}$. Attachment and detachment density plots ${ }^{93}$ are utilized to reveal where electron density is added and removed upon vertical excitation from ground to first-excited singlet states. 


\section{Acknowledgments}

The authors thank G. Schwaab, and R. Glaves for helpful discussions. This work is funded by the Deutsche Forschungsgemeinschaft (DFG, German Research Foundation) under Germany's Excellence Strategy - EXC 2033 - 390677874 - RESOLV. MH received financial support by the ERC Advanced Grant 695437 (THZCALORIMETRY). JS and MHG were supported by the Director, Office of Science, Office of Basic Energy Sciences, of the U.S. Department of Energy under Contract No. DE-AC02-05CH11231, and acknowledge additional support from CALSOLV. The authors declare no competing interests.

\section{Data and Materials Availability}

All data needed to evaluate the conclusions in the paper are present in the paper and/or the Supplementary Materials.

\section{References}

1 E. Pines, D. Huppert and N. Agmon, Geminate recombination in excited-state proton transfer reactions: Numerical solution of the Debye-Smoluchowski equation with backreaction and comparison with experimental results, J. Chem. Phys., 1988, 88, 5620-5630.

2 K. K. Smith, K. J. Kaufmann, D. Huppert and M. Gutman, Picosecond proton ejection: an ultrafast pH jump, Chem. Phys. Lett., 1979, 64, 522-527.

3 E. Pines and D. Huppert, Observation of geminate recombination in excited state proton transfer, J. Chem. Phys., 1986, 84, 3576-3577.

4 L. Genosar, B. Cohen and D. Huppert, Ultrafast Direct Photoacid-Base Reaction, J. Phys. Chem. A, 2000, 104, 6689-6698.

5 M. Rini, Real-Time Observation of Bimodal Proton Transfer in Acid-Base Pairs in Water, Science (80-. )., 2003, 301, 349-352.

6 N. Agmon, Elementary steps in excited-state proton transfer, J. Phys. Chem. A, 2005, 109, 1335.

7 O. F. Mohammed, J. Dreyer, B. Z. Magnes, E. Pines and E. T. J. Nibbering, Solvent-dependent photoacidity state of pyranine monitored by transient mid-infrared spectroscopy, ChemPhysChem, 2005, 6, 625-636.

8 S. K. Mondal, K. Sahu, S. Ghosh, P. Sen and K. Bhattacharyya, Excited-State Proton Transfer from Pyranine to Acetate in $\mathrm{\gamma}$-Cyclodextrin and Hydroxypropyl $\mathrm{\gamma}$-Cyclodextrin, J. Phys. Chem. A, 2006, 110, 13646-13652.

9 D. B. Spry, A. Goun and M. D. Fayer, Deprotonation dynamics and stokes shift of pyranine (HPTS), J. Phys. Chem. A, 2007, 111, 230-237.

10 W. Liu, F. Han, C. Smith and C. Fang, Ultrafast conformational dynamics of pyranine during excited state proton transfer in aqueous solution revealed by femtosecond stimulated Raman spectroscopy, J. Phys. Chem. B, 2012, 116, 10535-10550. 

Reaction Be beyond the Solvent-Control Limit?, J. Phys. Chem. B, 2015, 119, 2253-2262.

12 T. Kumpulainen, B. Lang, A. Rosspeintner and E. Vauthey, Ultrafast Elementary Photochemical Processes of Organic Molecules in Liquid Solution, Chem. Rev., 2017, 117, 10826-10939.

W. Liu, L. Tang, B. G. Oscar, Y. Wang, C. Chen and C. Fang, Tracking Ultrafast Vibrational Cooling during Excited-State Proton Transfer Reaction with Anti-Stokes and Stokes Femtosecond Stimulated Raman Spectroscopy, J. Phys. Chem. Lett., 2017, 8, 997-1003.

14 J. L. Pérez-Lustres, F. Rodriguez-Prieto, M. Mosquera, T. A. Senyushkina, N. P. Ernsting and S. A. Kovalenko, Ultrafast Proton Transfer to Solvent: Molecularity and Intermediates from Solvation- and Diffusion-Controlled Regimes, J. Am. Chem. Soc., 2007, 129, 5408-5418.

K. Ando and J. T. Hynes, Molecular Mechanism of $\mathrm{HCl}$ Acid Ionization in Water: Ab Initio Potential Energy Surfaces and Monte Carlo Simulations, J. Phys. Chem. B, 1997, 101, 1046410478.

A. Ansari, J. Berendzen, S. F. Bowne, H. Frauenfelder, I. E. Iben, T. B. Sauke, E. Shyamsunder and R. D. Young, Protein states and proteinquakes., Proc. Natl. Acad. Sci., 1985, 82, 50005004.

L. U. L. Brinkmann and J. S. Hub, Ultrafast anisotropic protein quake propagation after CO photodissociation in myoglobin, Proc. Natl. Acad. Sci., 2016, 113, 10565-10570.

M. Levantino, G. Schirò, H. T. Lemke, G. Cottone, J. M. Glownia, D. Zhu, M. Chollet, H. Ihee, A. Cupane and M. Cammarata, Ultrafast myoglobin structural dynamics observed with an X-ray free-electron laser, Nat. Commun., 2015, 6, 6772.

D. a. Schmidt, O. Birer, S. Funkner, B. P. Born, R. Gnanasekaran, G. W. Schwaab, D. M. Leitner and M. Havenith, Rattling in the Cage: Ions as Probes of Sub-picosecond Water Network Dynamics, J. Am. Chem. Soc., 2009, 131, 18512-18517.

S. Funkner, G. Niehues, D. a. Schmidt, M. Heyden, G. Schwaab, K. M. Callahan, D. J. Tobias and M. Havenith, Watching the Low-Frequency Motions in Aqueous Salt Solutions: The Terahertz Vibrational Signatures of Hydrated lons, J. Am. Chem. Soc., 2012, 134, 1030-1035.

21 S. Funkner, M. Havenith and G. Schwaab, Urea, a Structure Breaker? Answers from THz Absorption Spectroscopy, J. Phys. Chem. B, 2012, 116, 13374-13380.

22 H. Wirtz, S. Schäfer, C. Hoberg and M. Havenith, Differences in Hydration Structure Around Hydrophobic and Hydrophilic Model Peptides Probed by THz Spectroscopy, J. Infrared, Millimeter, Terahertz Waves, 2018, 39, 816-827.

23 G. Schwaab, F. Sebastiani and M. Havenith, Ion Hydration and Ion Pairing as Probed by $\mathrm{THz}$ Spectroscopy, Angew. Chemie Int. Ed., 2019, 58, 3000-3013.

24 D. Decka, G. Schwaab and M. Havenith, A THz/FTIR fingerprint of the solvated proton: evidence for Eigen structure and Zundel dynamics, Phys. Chem. Chem. Phys., 2015, 17, 1189811907.

25 J. Sun, G. Niehues, H. Forbert, D. Decka, G. Schwaab, D. Marx and M. Havenith, Understanding THz Spectra of Aqueous Solutions: Glycine in Light and Heavy Water, J. Am. Chem. Soc., 2014, 136, 5031-5038.

26 M. Heyden, J. Sun, S. Funkner, G. Mathias, H. Forbert, M. Havenith and D. Marx, Dissecting the THz spectrum of liquid water from first principles via correlations in time and space, Proc. Natl. Acad. Sci., 2010, 107, 12068-12073. 
27 P. Schienbein, G. Schwaab, H. Forbert, M. Havenith and D. Marx, Correlations in the SoluteSolvent Dynamics Reach Beyond the First Hydration Shell of Ions, J. Phys. Chem. Lett., 2017, 2373-2380.

28 S. Imoto, H. Forbert and D. Marx, Aqueous TMAO solutions as seen by theoretical THz spectroscopy: Hydrophilic: versus hydrophobic water, Phys. Chem. Chem. Phys., 2018, 20, 6146-6158.

A. Esser, H. Forbert, F. Sebastiani, G. Schwaab, M. Havenith and D. Marx, Hydrophilic Solvation Dominates the Terahertz Fingerprint of Amino Acids in Water, J. Phys. Chem. B, 2018, 122, 1453-1459.

30 F. Böhm, G. Schwaab and M. Havenith, Mapping Hydration Water around Alcohol Chains by THz Calorimetry, Angew. Chemie Int. Ed., 2017, 56, 9981-9985.

31 R. A. X. Persson, V. Pattni, A. Singh, S. M. Kast and M. Heyden, Signatures of Solvation Thermodynamics in Spectra of Intermolecular Vibrations, J. Chem. Theory Comput., 2017, 13, 4467-4481.

32 C. a. Schmuttenmaer, Exploring dynamics in the far-infrared with terahertz spectroscopy, Chem. Rev., 2004, 104, 1759-1779.

33 R. Ulbricht, E. Hendry, J. Shan, T. F. Heinz and M. Bonn, Carrier dynamics in semiconductors studied with time-resolved terahertz spectroscopy, Rev. Mod. Phys., 2011, 83, 543-586.

34 R. McElroy and K. Wynne, Ultrafast Dipole Solvation Measured in the Far Infrared, Phys. Rev. Lett., 1997, 79, 3078-3081.

35 G. Haran, W.-D. Sun, K. Wynne and R. M. Hochstrasser, Femtosecond far-infrared pump-probe spectroscopy: A new tool for studying low-frequency vibrational dynamics in molecular condensed phases, Chem. Phys. Lett., 1997, 274, 365-371.

36 F. Kadlec, C. Kadlec, P. Kužel, P. Slavíček and P. Jungwirth, Optical pump-terahertz probe spectroscopy of dyes in solutions: Probing the dynamics of liquid solvent or solid precipitate?, J. Chem. Phys., 2004, 120, 912-917.

37 E. Knoesel, M. Bonn, J. Shan and T. F. Heinz, Charge Transport and Carrier Dynamics in Liquids Probed by THz Time-Domain Spectroscopy, Phys. Rev. Lett., 2001, 86, 340-343.

38 J. Savolainen, F. Uhlig, S. Ahmed, P. Hamm and P. Jungwirth, Direct observation of the collapse of the delocalized excess electron in water, Nat Chem, 2014, 6, 697-701.

S. Ahmed, A. Pasti, R. J. Fernández-Terán, G. Ciardi, A. Shalit and P. Hamm, Aqueous solvation from the water perspective, J. Chem. Phys., 2018, 148, 234505.

40 U. Kaatze, The dielectric properties of water in its different states of interaction, J. Solution Chem., 1997, 26, 1049-1112.

41 M. Hauser, H.-P. Haar and U. K. A. Klein, Indirect Demonstration of the Coulomb Cage for Prototropic Dissociation, Berichte der Bunsengesellschaft für Phys. Chemie, 1977, 81, 27-30.

42 E. Bardez, B. T. Goguillon, E. Keh and B. Valeur, Dynamics of excited-state reactions in reversed micelles. 1. Proton transfer involving a hydrophilic fluorescent probe, J. Phys. Chem., 1984, 88, 1909-1913.

43 D. H. Huppert, A. Jayaraman, R. G. Maines, D. W. Steyert and P. M. Rentzepis, Effect of pressure on proton transfer rate in aqueous solutions: A picosecond study, J. Chem. Phys., $1984,81,5596-5600$. 
pp. 333-339.

45 U. Rivard, V. Thomas, A. Bruhacs, B. Siwick and R. Iftimie, Donor-Bridge-Acceptor Proton Transfer in Aqueous Solution, J. Phys. Chem. Lett., 2014, 5, 3200-3205.

46 B. J. Siwick and H. J. Bakker, On the Role of Water in Intermolecular Proton-Transfer Reactions, J. Am. Chem. Soc., 2007, 129, 13412-13420.

47 B. J. Siwick, M. J. Cox and H. J. Bakker, Long-Range Proton Transfer in Aqueous Acid-Base Reactions †, J. Phys. Chem. B, 2008, 112, 378-389.

48 D. Pines, E. T. J. Nibbering and E. Pines, Monitoring the Microscopic Molecular Mechanisms of Proton Transfer in Acid-base Reactions in Aqueous Solutions, Isr. J. Chem., 2015, 55, 1240 1251.

49 P. Leiderman, L. Genosar and D. Huppert, Excited-state proton transfer: Indication of three steps in the dissociation and recombination process, J. Phys. Chem. A, 2005, 109, 5965-5977.

50 R. Simkovitch, G. G. Rozenman and D. Huppert, A fresh look into the time-resolved fluorescence of 8-hydroxy-1,3,6-pyrenetrisulfonate with the use of the fluorescence upconversion technique, J. Photochem. Photobiol. A Chem., 2017, 344, 15-27.

51 D. B. Spry and M. D. Fayer, Charge redistribution and photoacidity: Neutral versus cationic photoacids, J. Chem. Phys., 2008, 128, 084508.

52 T.-H. Tran-Thi, T. Gustavsson, C. Prayer, S. Pommeret and J. T. Hynes, Primary ultrafast events preceding the photoinduced proton transfer from pyranine to water, Chem. Phys. Lett., 2000, 329, 421-430.

53 J. T. Hynes, T.-H. Tran-Thi and G. Granucci, Intermolecular photochemical proton transfer in solution: new insights and perspectives, J. Photochem. Photobiol. A Chem., 2002, 154, 3-11.

54 D. B. Spry, A. Goun, C. B. Bell and M. D. Fayer, Identification and properties of the La1 and Lb1 states of pyranine, J. Chem. Phys., 2006, 125, 144514.

55 R. Gepshtein, P. Leiderman, L. Genosar and D. Huppert, Testing the Three Step Excited State Proton Transfer Model by the Effect of an Excess Proton, J. Phys. Chem. A, 2005, 109, 96749684.

56 O. F. Mohammed, D. Pines, E. T. J. Nibbering and E. Pines, Base-Induced Solvent Switches in Acid-Base Reactions, Angew. Chemie Int. Ed., 2007, 46, 1458-1461.

57 T. Kumpulainen, A. Rosspeintner, B. Dereka and E. Vauthey, Influence of Solvent Relaxation on Ultrafast Excited-State Proton Transfer to Solvent, J. Phys. Chem. Lett., 2017, 8, 4516-4521.

58 C. Hoberg, P. Balzerowski and M. Havenith, Integration of a rapid scanning technique into $\mathrm{THz}$ time-domain spectrometers for nonlinear THz spectroscopy measurements, AIP Adv., 2019, 9, 035348.

59 M. Rosenberg, C. Dahlstrand, K. Kilså and H. Ottosson, Excited State Aromaticity and Antiaromaticity: Opportunities for Photophysical and Photochemical Rationalizations, Chem. Rev., 2014, 114, 5379-5425.

60 J. Oh, Y. M. Sung, Y. Hong and D. Kim, Spectroscopic Diagnosis of Excited-State Aromaticity: Capturing Electronic Structures and Conformations upon Aromaticity Reversal, Acc. Chem. Res., 2018, 51, 1349-1358.

61 C.-H. Wu, L. J. Karas, H. Ottosson and J. I. C. Wu, Excited-state proton transfer relieves antiaromaticity in molecules, Proc. Natl. Acad. Sci., 2019, 116, 20303-20308. 

Observed by Stark Spectroscopy, J. Phys. Chem. A, 2008, 112, 10244-10249.

63 W. Liu, Y. Wang, L. Tang, B. G. Oscar, L. Zhu and C. Fang, Panoramic portrait of primary molecular events preceding excited state proton transfer in water, Chem. Sci., 2016, 7, 54845494.

M. G. Chiariello and N. Rega, Exploring Nuclear Photorelaxation of Pyranine in Aqueous Solution: an Integrated Ab-Initio Molecular Dynamics and Time Resolved Vibrational Analysis Approach, J. Phys. Chem. A, 2018, 122, 2884-2893.

E. W. Castner and M. Maroncelli, Solvent dynamics derived from optical Kerr effect, dielectric dispersion, and time-resolved stokes shift measurements: an empirical comparison, J. Mol. Liq., 1998, 77, 1-36.

G. Niehues, A. L. Kaledin, J. M. Bowman and M. Havenith, Driving of a Small Solvated Peptide in the IR and THz Range-A Comparative Study of Energy Flow, J. Phys. Chem. B, 2012, 116, 10020-10025.

T. D. Krueger, S. A. Boulanger, L. Zhu, L. Tang and C. Fang, Discovering a rotational barrier within a charge-transfer state of a photoexcited chromophore in solution, Struct. Dyn., 2020, 7, 024901.

U. Kaatze, Dielectric relaxation of H2O/D2O mixtures, Chem. Phys. Lett., 1993, 203, 1-4.

R. Mills, Self-diffusion in normal and heavy water in the range 1-45.deg., J. Phys. Chem., 1973, 77, 685-688.

R. Simkovitch, D. Pines, N. Agmon, E. Pines and D. Huppert, Reversible Excited-State Proton Geminate Recombination: Revisited, J. Phys. Chem. B, 2016, 120, 12615-12632.

71 M. Heyden and D. J. Tobias, Spatial Dependence of Protein-Water Collective Hydrogen-Bond Dynamics, Phys. Rev. Lett., 2013, 111, 218101.

72 V. Conti Nibali and M. Havenith, New Insights into the Role of Water in Biological Function: Studying Solvated Biomolecules Using Terahertz Absorption Spectroscopy in Conjunction with Molecular Dynamics Simulations, J. Am. Chem. Soc., 2014, 136, 12800-12807.

73 D. C. Elton and M. Fernández-Serra, The hydrogen-bond network of water supports propagating optical phonon-like modes, Nat. Commun., 2016, 7, 10193.

74 F. Yang, T. Atay, C. H. Dang, T. J. Grimsley, S. Che, J. Ma, Q. Zhang, A. V. Nurmikko and H. J. Maris, Study of phonon propagation in water using picosecond ultrasonics, J. Phys. Conf. Ser., 2007, 92, 012024.

75 B. Bagchi, D. W. Oxtoby and G. R. Fleming, Theory of the time development of the stokes shift in polar media, Chem. Phys., 1984, 86, 257-267.

76 B. Bagchi and B. Jana, Solvation dynamics in dipolar liquids, Chem. Soc. Rev., 2010, 39, 19361954.

77 F. Sebastiani, C. Y. Ma, S. Funke, A. Bäumer, D. Decka, C. Hoberg, A. Esser, H. Forbert, G. Schwaab, D. Marx and M. Havenith, Probing Local Electrostatics of Glycine in Aqueous Solution by THz Spectroscopy, Angew. Chemie Int. Ed., 2021, 60, 3768-3772.

78 P. Cimino, U. Raucci, G. Donati, M. G. Chiariello, M. Schiazza, F. Coppola and N. Rega, On the different strength of photoacids, Theor. Chem. Acc., 2016, 135, 117.

79 P. Verma, A. Rosspeintner, B. Dereka, E. Vauthey and T. Kumpulainen, Broadband fluorescence reveals mechanistic differences in excited-state proton transfer to protic and 
aprotic solvents, Chem. Sci., 2020, 11, 7963-7971.

F. Novelli, J. W. M. Chon and J. A. Davis, Terahertz thermometry of gold nanospheres in water, Opt. Lett., 2016, 41, 5801.

81 C. Hoberg, T. Ockelmann, J. Shee, P. Balzerowski, D. DasMahanta, F. Novelli, M. Head-Gordon and M. Havenith, in 2020 45th International Conference on Infrared, Millimeter, and Terahertz Waves (IRMMW-THz), IEEE, 2020.

D. J. Cook and R. M. Hochstrasser, Intense terahertz pulses by four-wave rectification in air, Opt. Lett., 2000, 25, 1210.

X. Xie, J. Dai and X. C. Zhang, Coherent control of THz wave generation in ambient air, Phys. Rev. Lett., 2006, 96, 1-4.

84 T. Bartel, P. Gaal, K. Reimann, M. Woerner and T. Elsaesser, Generation of single-cycle THz transients with high electric-field amplitudes, Opt. Lett., 2005, 30, 2805.

E. H. G. Backus, K. J. Tielrooij, M. Bonn and H. J. Bakker, Probing ultrafast temperature changes of aqueous solutions with coherent terahertz pulses, Opt. Lett., 2014, 39, 1717-1720.

86 C. Spies, B. Finkler, N. Acar and G. Jung, Solvatochromism of pyranine-derived photoacids, Phys. Chem. Chem. Phys., 2013, 15, 19893.

87 Y. Shao, Z. Gan, E. Epifanovsky, A. T. B. Gilbert, M. Wormit, J. Kussmann, A. W. Lange, A. Behn, J. Deng, X. Feng, D. Ghosh, M. Goldey, P. R. Horn, L. D. Jacobson, I. Kaliman, R. Z. Khaliullin, T. Kuś, A. Landau, J. Liu, E. I. Proynov, Y. M. Rhee, R. M. Richard, M. A. Rohrdanz, R. P. Steele, E. J. Sundstrom, H. L. Woodcock, P. M. Zimmerman, D. Zuev, B. Albrecht, E. Alguire, B. Austin, G. J. O. Beran, Y. A. Bernard, E. Berquist, K. Brandhorst, K. B. Bravaya, S. T. Brown, D. Casanova, C.-M. Chang, Y. Chen, S. H. Chien, K. D. Closser, D. L. Crittenden, M. Diedenhofen, R. A. DiStasio, H. Do, A. D. Dutoi, R. G. Edgar, S. Fatehi, L. Fusti-Molnar, A. Ghysels, A. GolubevaZadorozhnaya, J. Gomes, M. W. D. Hanson-Heine, P. H. P. Harbach, A. W. Hauser, E. G. Hohenstein, Z. C. Holden, T.-C. Jagau, H. Ji, B. Kaduk, K. Khistyaev, J. Kim, J. Kim, R. A. King, P. Klunzinger, D. Kosenkov, T. Kowalczyk, C. M. Krauter, K. U. Lao, A. D. Laurent, K. V. Lawler, S. V. Levchenko, C. Y. Lin, F. Liu, E. Livshits, R. C. Lochan, A. Luenser, P. Manohar, S. F. Manzer, S.P. Mao, N. Mardirossian, A. V. Marenich, S. A. Maurer, N. J. Mayhall, E. Neuscamman, C. M. Oana, R. Olivares-Amaya, D. P. O'Neill, J. A. Parkhill, T. M. Perrine, R. Peverati, A. Prociuk, D. R. Rehn, E. Rosta, N. J. Russ, S. M. Sharada, S. Sharma, D. W. Small, A. Sodt, T. Stein, D. Stück, Y.C. Su, A. J. W. Thom, T. Tsuchimochi, V. Vanovschi, L. Vogt, O. Vydrov, T. Wang, M. A. Watson, J. Wenzel, A. White, C. F. Williams, J. Yang, S. Yeganeh, S. R. Yost, Z.-Q. You, I. Y. Zhang, X. Zhang, Y. Zhao, B. R. Brooks, G. K. L. Chan, D. M. Chipman, C. J. Cramer, W. A. Goddard, M. S. Gordon, W. J. Hehre, A. Klamt, H. F. Schaefer, M. W. Schmidt, C. D. Sherrill, D. G. Truhlar, A. Warshel, X. Xu, A. Aspuru-Guzik, R. Baer, A. T. Bell, N. A. Besley, J.-D. Chai, A. Dreuw, B. D. Dunietz, T. R. Furlani, S. R. Gwaltney, C.-P. Hsu, Y. Jung, J. Kong, D. S. Lambrecht, W. Liang, C. Ochsenfeld, V. A. Rassolov, L. V. Slipchenko, J. E. Subotnik, T. Van Voorhis, J. M. Herbert, A. I. Krylov, P. M. W. Gill and M. Head-Gordon, Advances in molecular quantum chemistry contained in the Q-Chem 4 program package, Mol. Phys., 2015, 113, 184-215.

P. J. Stephens, F. J. Devlin, C. F. Chabalowski and M. J. Frisch, Ab Initio Calculation of Vibrational Absorption and Circular Dichroism Spectra Using Density Functional Force Fields, J. Phys. Chem., 1994, 98, 11623-11627.

F. Weigend and R. Ahlrichs, Balanced basis sets of split valence, triple zeta valence and quadruple zeta valence quality for $\mathrm{H}$ to $\mathrm{Rn}$ : Design and assessment of accuracy, Phys. Chem. Chem. Phys., 2005, 7, 3297. 
of molecules in solution with the C-PCM solvation model, J. Comput. Chem., 2003, 24, 669681.

91 E. Runge and E. K. U. Gross, Density-Functional Theory for Time-Dependent Systems, Phys. Rev. Lett., 1984, 52, 997-1000.

92 M. E. Casida, in Recent Advances In Density Functional Methods: (Part I), 1995, pp. 155-192.

93 M. Head-Gordon, A. M. Grana, D. Maurice and C. A. White, Analysis of Electronic Transitions as the Difference of Electron Attachment and Detachment Densities, J. Phys. Chem., 1995, 99, 14261-14270. 